\title{
Current-Driven Kink Instability in Relativistic Jets
}

\author{
Yosuke Mizuno $^{1}$, Philip E. Hardee ${ }^{2}$, Yuri Lyubarsky ${ }^{3}$, \\ and Ken-Ici Nishikawa ${ }^{1}$ \\ ${ }^{1}$ CSPAR, The University of Alabama in Huntsville, \\ 320 Sparkman Drive, NSSTC, Huntsville, AL 35805, USA \\ email: mizuno@cspar.uah.edu \\ ${ }^{2}$ Dept. Physics and Astronomy, University of Alabama, Tuscaloosa, AL 35487, USA \\ ${ }^{3}$ Physics Department, Ben-Gurion University, Beer-Sheva 84105, Israel
}

\begin{abstract}
We have investigated the development of current-driven (CD) kink instability in relativistic jets via 3D RMHD simulations. In this investigation a static force-free equilibrium helical magnetic field configuration is considered in order to study the influence of the initial configuration on the linear and nonlinear evolution of the instability. We found that the initial configuration is strongly distorted but not disrupted by the CD kink instability. The linear growth and nonlinear evolution of the CD kink instability depends moderately on the radial density profile and strongly on the magnetic pitch profile. Kink amplitude growth in the nonlinear regime for decreasing magnetic pitch leads to a slender helically twisted column wrapped by magnetic field. On the other hand, kink amplitude growth in the nonlinear regime nearly ceases for increasing magnetic pitch.
\end{abstract}

Keywords. relativistic jets, instabilities, MHD

\section{Introduction}

Relativistic jets occur in active galactic nuclei (AGN), occur in microquasars ( $\mu$ QSOs), and are thought responsible for the gamma-ray bursts (GRB). The most promising mechanism for producing relativistic jets involves magnetohydrodynamic acceleration from an accretion disk around a black hole (Blandford \& Payne 1982), and/or involves the extraction of energy from a rotating black hole (Blandford \& Znajek 1977).

GRMHD simulations with a spinning black hole indicate jet production with a magnetically dominated high Lorentz factor spine with $v \sim c$, and a matter dominated sheath with $v \gtrsim c / 2$ possibly embedded in a lower speed, $v \ll c$, disk/coronal wind involving helically twisted magnetic fields (e.g., Hawley \& Krolik 2006; McKinney 2006; Hardee et al. 2007).

In configurations with a strong toroidal magnetic field the current driven (CD) kink mode is unstable. This instability excites large-scale helical motions that can strongly distort or even disrupt the system. For static cylindrical force-free equilibria, the wellknown Kruskal-Shafranov criterion states that the instability develops if the length of the column, $\ell$, is long enough for the field lines to go around the cylinder at least once (Bateman 1978): $\left|B_{p} / B_{\phi}\right|<\ell / 2 \pi R$. However, rotation and shear motions could significantly affect the instability criterion. For relativistic force-free configurations, the linear instability criteria have been studied by a number of researchers (e.g., Begelman 1998; Lyubarskii 1999; Narayan et al. 2009).

The linear mode analysis provides conditions for the instability but says little about the impact the instability has on the system. To evaluate the impact of the potentially disruptive kink mode found from a linear analysis the instability must be followed into the non-linear regime. 
In this work, we study the kink instability in relativistic systems. By relativistic we mean not only relativistically moving systems but any with magnetic energy density comparable to or greater than the plasma energy density, including the rest mass energy. In particular, we present 3D results of the CD kink instability of a static plasma column (Mizuno et al. 2009).

\section{Numerical Setup and Results}

In order to study time evolution of the CD kink instability in the relativistic MHD (RMHD) regime, we use the 3D GRMHD code "RAISHIN" in Cartesian coordinates (Mizuno et al. 2006). For our simulations we will choose a force-free helical magnetic field as the initial configuration. A pitch profile parameter $\alpha$ determines the radial profile of the magnetic pitch $P=R B_{z} / B_{\phi}$, and provides a measure of the twist of the magnetic field lines. If the pitch profile parameter $0.5<\alpha<1$, the magnetic pitch increases with radius. If $\alpha>1$, the magnetic pitch decreases. When $\alpha=1$, the magnetic pitch is constant. This configuration is the same as that used in previous non-relativistic work (Appl et al. 2000; Baty 2005).

In our simulations we consider a low gas pressure medium with constant $p=p_{0}=$ $0.02 \rho_{0} c^{2}$ for the equilibrium state, and a non-uniform density profile decreasing proportional to the magnetic field strength, $\rho=\rho_{1} B^{2}$ with $\rho_{1}=10.0 \rho_{0}$. The magnetic field amplitude is $B_{0}=0.4 \sqrt{4 \pi \rho_{0} c^{2}}$ and leads to a low plasma- $\beta$ near the axis. In order to investigate the effect of different radial pitch profiles, we perform simulations with: constant pitch, $\alpha=1$, increasing pitch, $\alpha=0.75$, and decreasing pitch $\alpha=2.0$.

The simulation grid is periodic along the axial direction. The grid is a Cartesian $(x, y, z)$ box of size $4 L \times 4 L \times 2 L$ with grid resolution of $\Delta L=L / 40$, where $L$ is a simulation scale unit. We impose outflow boundary conditions on the transverse boundaries at $x=$ $y= \pm 2 L$.

The initial MHD equilibrium configuration is perturbed by a small radial velocity component with profile given by $v_{R}=\delta v \exp \left(-R / R_{a}\right) \cos (m \theta) \sin \left(2 \pi n z / L_{z}\right)$ with $\delta v=$ $0.01 c$ and $R_{a}=0.5 L$. We choose $m=1$ and $n=1$ in the above formula. This is identical to imposing $(m, n)=(-1,-1)$, because of the symmetry between $(m, n)$ and $(-m,-n)$ pairs. A detailed description of numerical set-up can be found in Mizuno et al. (2009).

As an indicator of the growth of the CD kink instability we use the volume-averaged kinetic energy transverse to the $z$-axis $\left(E_{k i n, x y}\right)$. The quantity $E_{k i n, x y}$ allows determination of different evolutionary stages, e.g., initial exponential (linear growth phase) growth and subsequent non-linear evolution. In all cases, the initial growth regime is characterized by an exponential increase in $E_{k i n, x y}$ by several orders of magnitude to a maximum amplitude followed by a slow decline in the non-linear regime. The increasing pitch case ( $\alpha=0.75)$ grows more slowly and reaches a maximum at a later time with a smaller value for $E_{k i n, x y}$ than the constant pitch case $(\alpha=1.0)$. On the other hand, the decreasing pitch case $(\alpha=2.0)$ grows more rapidly and reaches a maximum at an earlier time with a larger value for $E_{k i n, x y}$ than the constant pitch case. Although the transition time from linear to non-linear evolution is different for each pitch case, the maximum radial velocity is almost the same at transition. The different growth rates as a function of the radial pitch profile are consistent with a non-relativistic linear analysis (Appl et al. 2000).

Figure 1 shows density isosurfaces with magnetic field lines for the constant pitch, increasing pitch and decreasing pitch cases for the non-uniform density profile at time $t_{A}=96\left(L / 8 v_{A 0}\right)$. Displacement of the initial force-free helical magnetic field leads to a helically twisted magnetic filament winded around the density isosurface. In the constant pitch case the radial displacement of the high density region slows significantly at longer 
times. Continuing outwards radial motion is confined to a lower density sheath around the high density core.

(a) Constant Pitch

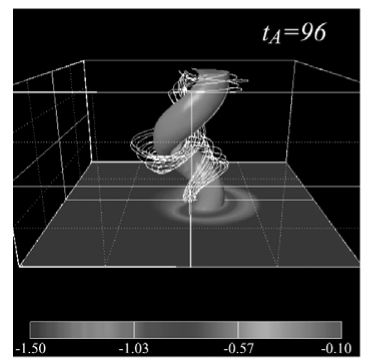

(b) Increasing Pitch

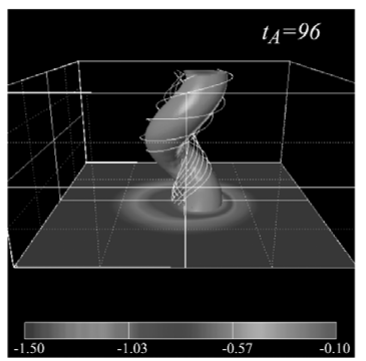

(c) Decreasing Pitch



Figure 1. 3D density isosurface for (a) constant pitch, (b) increasing pitch, and (c) decreasing pitch cases. Colors show the logarithm of the density with (white) magnetic field lines.

The 3D density structure of the increasing pitch case looks similar to that of the constant pitch case. The CD kink instability initially grows exponentially. A transverse density slice shows little outwards motion of the high density region in the non-linear stage similar to the constant pitch case; however, there is little outwards motion in the low density sheath surrounding the high density region. This result is somewhat different from the constant pitch case and suggests a significant reduction in kink amplitude growth. Results from the decreasing pitch case are very different. Figure $2 \mathrm{c}$ shows a more slender helical density structure wrapped by the magnetic field. While the density cross section is similar to that of the constant pitch case, radial motion continues in the non-linear stage to the end of the simulation.

\section{Acknowledgements}

This work is supported in part by NSF awards AST-0506719, AST-0506666, AST0908010, AST-0908040, NASA awards NNG05GK73G, NNX07AJ88G, NNX08AG83G, and US-Israeli BSF 2006170. The simulations were performed on the NAS Division Columbia Supercomputer at the NASA Ames Research Center and the Altix3700 BX2 at YITP in Kyoto University.

\section{References}

Appl, S., Lery, T., \& Baty, H. 2000 A $\& A 355,818$

Bateman, G. 1978, MHD Instabilities (Cambridge, MA: MIT Press), p. 270

Baty, H. 2005 A\&SA 430, 9

Begelman, M. C. 1998 ApJ 493, 291

Blandford, R. D. \& Payne, D. G. 1982, MNRAS 199, 883

Blandford, R. D. \& Znajek, R. L. 1977, MNRAS 179, 433

Hardee, P., Mizuno, Y., \& Nishikawa, K.-I. 2007 Ap\&SS 311, 283

Hawley, J. F. \& Krolik, J. H. 2006, ApJ 641, 103

Lyubarskii, Y. E., 1999 MNRAS 208, 1006

McKinney, J. C. 2006, MNRAS 368, 1561.

Mizuno, Y., Lyubarsky, Y., Nishikawa, K.-I., \& Hardee, P. E. 2009 ApJ 700, 684

Mizuno, Y., Nishikawa, K.-I., Koide, S., Hardee, P., \& Fishman, G. J. 2006 ArXiv Astrophysics e-prints 0609004

Narayan, R., Li, J., \& Tchekhovskoy, A. 2009, ApJ 697, 1681 